\section{A genome-wide CRISPR screen identifies regulators of MAPK and MTOR pathways that mediate resistance to sorafenib in acute myeloid leukemia}

\author{
Alisa Damnernsawad, ${ }^{1,2}$ Daniel Bottomly, ${ }^{3}$ Stephen E. Kurtz, ${ }^{4}$ Christopher A. \\ Eide, ${ }^{4}$ Shannon K. McWeeney, ${ }^{3}$ Jeffrey W. Tyner ${ }^{1,4}$ and Tamilla Nechiporuk ${ }^{1}$ \\ ${ }^{1}$ Department of Cell, Developmental \& Cancer Biology, Knight Cancer Institute, Oregon \\ Health \& Science University, Portland, OR, USA; ${ }^{2}$ Department of Biology, Faculty of \\ Science, Mahidol University, Bangkok, Thailand; ${ }^{3}$ Division of Bioinformatics and \\ Computational Biology, Knight Cancer Institute, Oregon Health \& Science University, \\ Portland, OR, USA and ${ }^{4}$ Division of Hematology and Medical Oncology, Knight Cancer \\ institute, Oregon Health \& Science University, Portland, OR, USA
}

\section{ABSTRACT}

$\mathrm{D}$ rug resistance impedes the long-term effect of targeted therapies in acute myeloid leukemia (AML), necessitating the identification of mechanisms underlying resistance. Approximately 25\% of AML patients carry FLT3 mutations and develop post-treatment insensitivity to FLT3 inhibitors, including sorafenib. Using a genomewide CRISPR screen, we identified LZTR1, NF1, TSC1 and TSC2, negative regulators of the MAPK and MTOR pathways, as mediators of resistance to sorafenib. Analyses of ex vivo drug sensitivity assays in samples from patients with FLT3-ITD AML revealed that lower expression of LZTR1, NF1, and TSC2 correlated with sensitivity to sorafenib. Importantly, MAPK and/or MTOR complex 1 (MTORC1) activity was upregulated in AML cells made resistant to several FLT3 inhibitors, including crenolanib, quizartinib, and sorafenib. These cells were sensitive to MEK inhibitors, and the combination of FLT3 and MEK inhibitors showed enhanced efficacy, suggesting the effectiveness of such treatment in AML patients with FLT3 mutations and those with resistance to FLT3 inhibitors.

\section{Introduction}

Acute myeloid leukemia (AML), a rapidly progressing hematologic malignancy, is caused by the impaired differentiation and subsequent proliferation of hematopoietic progenitor cells. AML is characterized by cytogenetic heterogeneity and numerous recurrent genetic lesions. ${ }^{1.5}$ The Fms-related tyrosine kinase 3 (FLT3) receptor tyrosine kinase is normally expressed on hematopoietic stem and progenitor cells and functions in promoting cell proliferation and survival as well as normal development of these cells. ${ }^{6-8}$ FLT3 activating mutations occur in approximately $25 \%$ of AML patients, either by internal tandem duplications (FLT3-ITD) or point mutations in the tyrosine kinase domain, ${ }^{4,-12}$ stimulating AML cell proliferation and survival. These mutations are associated with poor outcomes including an enhanced risk of relapse. ${ }^{6,713}$ The high frequency and adverse effects of FLT3 mutations have prompted the development of small-molecule inhibitors targeting FLT3.

Among the FLT3 tyrosine kinase inhibitors that have been developed, several have provided encouraging results in clinical trials, ${ }^{7,14,15}$ and two in particular, midostaurin and gilteritinib, have been approved for FLT3-mutant AML. ${ }^{16-18}$ Nevertheless, all the FLT3 inhibitors developed to date lack long-term, durable clinical efficacy because of the development of resistance. Point mutations within the kinase domain of FLT3, such as variants in residues D835 and F691, cause resistance to type II FLT3 inhibitors (quizartinib and sorafenib) in vitro as well as in relapsed/refractory patients. ${ }^{19-21}$ While patients with tyrosine kinase domain mutations develop resistance to type II inhibitors, they are sensitive to type I
Haematologica 2022

Volume 107(1):77-85

\section{Correspondence:}

TAMILLA NECHIPORUK

nechipot@ohsu.edu

JEFFREY W. TYNER

tynerj@ohsu.edu

Received: May 3, 2020.

Accepted: December 21, 2020.

Pre-published: December 30, 2020.

https://doi.org/10.3324/haematol.2020.257964

(C)2022 Ferrata Storti Foundation

Material published in Haematologica is covered by copyright. All rights are reserved to the Ferrata Storti Foundation. Use of published material is allowed under the following terms and conditions:

https://creativecommons.org/licenses/by-nc/4.0/legalcode. Copies of published material are allowed for personal or internal use. Sharing published material for non-commercial purposes is subject to the following conditions:

https://creativecommons.org/licenses/by-nc/4.0/legalcode, sect. 3. Reproducing and sharing published material for commercial purposes is not allowed without permission in writing from the publisher. 
inhibitors, even though the responses are transient. ${ }^{21}$ Diverse mutations underlie resistance in $\mathrm{AML}$ patients to the type I inhibitor, crenolanib, including rare mutations at the gatekeeper region of FLT3, as well as NRAS and IDH2 mutations in FLT3-independent subclones, and TET2 and IDH1 mutations in FLT3 mutant clones in nonresponding patients. ${ }^{22}$ Single-cell DNA sequencing of specimens from patients relapsing after treatment with a novel type I inhibitor, gilteritinib, revealed shifts in clonal architecture to select for secondary mutations in NRAS, KRAS, IDH2, or BCR-ABL1, either in the context of FLT3ITD or FLT3 wild-type clones. ${ }^{18}$ Aberrant activation of ERK either extrinsically through the bone marrow microenvironment or intrinsically in a cell-autonomous manner has been implicated in FLT3 resistance in AML. ${ }^{23,24}$ Upregulation of the RAS/RAF/ERK pathway has been observed after treatment with FLT3 tyrosine kinase inhibitors in AML cell lines and AML patients' bone marrow samples. ${ }^{23,25}$ Signaling through JAK/STAT5 mediated by granulocyte-macrophage colony-stimulating factor and interleukin-3 allows AML cells to survive FLT3 inhibitor treatment. ${ }^{26}$ Activation of the phosphatidylinositol-3 kinase (PI3K)/mammalian target of rapamycin (MTOR) pathway has also been demonstrated to promote resistance to a FLT3 inhibitor. ${ }^{27}$

Sorafenib, a multi-kinase inhibitor targeting not only FLT3 but also RAF, VEGFR, FGFR, KIT and RET, ${ }^{28}$ has been evaluated in combination with azacytidine in AML patients with FLT3-ITD, who had an overall response rate of $46 \% .{ }^{29}$ The combination of sorafenib and standard-ofcare chemotherapy extended event-free survival in patients younger than 60 years old.$^{30}$ Data from a phase I trial showed that patients harboring FLT3-ITD who were treated with allogeneic hematopoietic stem cell transplantation had a 1-year progression-free survival rate of $85 \%$ and a 1 -year overall survival rate of $95 \% .^{31}$

To identify mechanisms of resistance to sorafenib we used a genome-wide CRISPR (clusters of regularly interspaced short palindromic repeats) knockout screen to search for genes whose loss-of-function variants can promote FLT3 inhibitor-sensitive AML cells to survive in the presence of sorafenib. To confirm that aberrant signaling in the identified pathways renders cells insensitive to FLT3 inhibitors, we established AML cells resistant to both type I and type II FLT3 inhibitors. Our CRISPR screen identified genes in the MTOR and mitogen-activated protein kinase (MAPK) pathways that modulate sensitivity to sorafenib. Activities of MTOR and MAPK pathways were upregulated in cells with acquired resistance, and these cells were sensitive to MEK inhibitors supporting the role of aberrant downstream MAPK signaling in resistance to FLT3 inhibitors. We found the combination of FLT3 and MEK inhibitors had synergistic efficacy in both FLT3 inhibitor-sensitive and -resistant AML cells as well as in samples from AML patients. In summary, our work identified several negative regulators of MTOR and MAPK signaling pathways, LZTR1, TSC1/2, NPRL2, NF1, not previously associated with AML, as modulators of sensitivity to sorafenib. We show that aberrations in MTOR and MAPK pathways are important mechanisms of resistance to sorafenib as well as other FLT3 inhibitors in AML and suggest that the combination of FLT3 inhibitors and MEK inhibitors could be useful for the treatment of FLT3 inhibitor-resistant AML.

\section{Methods}

\section{Cell lines}

Human MOLM13 cells were obtained from the Sanger Institute Cancer Cell Line Panel. All cell lines used in this study were authenticated at the OHSU DNA Services Core facility. Cell lines were maintained in $20 \%$ fetal bovine serum, RPMI medium, supplemented with glutamine, penicillin/streptomycin and an antimycotic. All cell lines were tested for Mycoplasma on a monthly schedule.

\section{Lentivirus production and transduction}

HEK293T cells were transfected using Lipofectamine-2000 (Invitrogen) with single transfer vectors in combination with packaging plasmids, psPax2 (Addgene, \#12260) and VSVG (Invitrogen). Supernatants were collected, filtered through $0.45 \mu \mathrm{M}$ filters and used for transduction as described previously.

\section{The CRISPR/Cas9 library screen and CRISPR/Cas9 gene inactivation by individual sgRNA}

Cas9-expressing cells were generated using Cas9Blst (Addgene, \#52962). Loss-of-function screens were performed using pooled human genome-wide single-guide (sg)RNA libraries, the Y. Kosuke library, ${ }^{33}$ purchased from Addgene (\#67989), as described previously, ${ }^{32}$ which targets 18,010 genes with 90,709 sgRNA (average of 5 guides per gene). High-titer lentivirus was generated using standard calcium phosphate precipitation procedures in HEK293T cells. Viral supernatant was concentrated and the titer determined using a viral titration kit (ABM good, Canada). One hundred million cells were used for viral transduction at a multiplicity of infection (MOI) of 0.3 , selected with puromycin for 5-7 days to ensure stable viral integration. Individual genes were inactivated by cloning sgRNA into plentiCRISPRV2 (Addgene, \#52961) according to the manufacturer's suggestions. The following sgRNA were used in the study: LZTR1: 5' CCCATAGACGACGGCCGAG 3', NF1: 5'CATATCAGTCTGTGGGATC 3', TSC1, 5'ACGTCGTTGTCCTCACAAC 3', TSC2: 5' TTGATGCGCACGGCGCCTC 3', NPRL2: 5' GAACCCATCAATGTAGGGC 3', DEPDC 5' GACTGTGACTCAAGTGTTCC and 5' TGTTAATGTCGTAGACCCTA, TBC1D7 5' GTATCGTASAGGAGCAGTACT. Sequencing data were deposited to GEO with, accession number GSE138343.

\section{Drug sensitivity assay}

Small-molecule inhibitors, purchased from LC Laboratories Inc. (Woburn, MA, USA) and Selleck Chemicals (Houston, TX, USA), were reconstituted in dimethylsulfoxide (DMSO). Cells were seeded at 1,000 cells/well in a 384-well plate in $50 \mu \mathrm{L}$ medium (RPMI-1640 supplemented with fetal bovine serum [15\%], L-glutamine, penicillin-streptomycin and an antimycotic) with different concentrations of drugs and cultured for $72 \mathrm{~h}$. For the drug sensitivity assay, $5 \mathrm{uL}$ of MTS reagent (CellTiter96 $\mathrm{AO}_{\text {wew }}$ One; Promega Madison, WI, USA) were added to each well and incubated for 4 h. Optical density was measured at $490 \mathrm{~nm}$. Relative cell viability was calculated by normalizing the readings to those of untreated control wells. Prism software (GraphPad) was used to produce non-linear fitting and determine the response to the drug, the half maximal inhibitory concentration (ICs) and the area under the curve (AUC).

\section{Immunoblot analysis}

Whole cell protein lysates were prepared using cell lysis buffer (Cell Signaling Technologies), $1 \mathrm{mM}$ phenylmethylsulfonyl fluoride, proteasome (Roche) and a phosphatase inhibitor cocktail (Sigma-Aldrich). Proteins were resolved on $4-15 \%$ gradient gels 
(Biorad), transferred onto polyvinylidene fluoride membranes (Amersham), and subjected to immunoblotting using primary antibodies from Cell Signaling Technologies: p44/42 MAPK (ERK1/2; \#9102), phospho-ERK1/2 (phospho-p44/42 MAPK Thr202/Tyr204; \#4376), AKT (\#9272), phospho-AKT (Ser473; \#4060), TSC1 (\#6935), phospho-TSC2 (Ser664; \#40729), phosphoTSC2 (Tyr1571; \#3614), TSC2 (\#4308), phospho-mTOR (Ser2481; \#2974), phospho-mTOR (Ser2448; \#2971), mTOR (\#2983), NF1 (\#14623), MEK (\#9122S), phosphor-MEK (\#9154), vinculin (\#4650); from ThermoFisher Scientific: GAPDH (\#AM4300); from Millipore: anti-pan_Ras (clone RAS 10 MABS195) from Sigma: LZTR1 (HPA071248). Corresponding horseradish perodixase-conjugated secondary antibodies (Promega) were used for chemiluminescent detection.

\section{Biostatistical analysis}

The bioinformatics pipeline for analyzing CRISPR library sequences was MAGeCK (model-based analysis of genome-wide CRISPR-Cas9 knockout). ${ }^{34}$ The hits were prioritized according to a previously described tiering structure..$^{32}$ Briefly, tier 1 represents hits having a log, fold change $\geq 2,75 \%$ of sgRNA per gene present and concordance among sgRNA per gene $\geq 75 \%$; tier 2 hits have a $\log _{2}$ fold change $\geq 2$ and concordance among sgRNA per gene of $100 \%$; tier 3 hits have a log, fold change $\geq 1$ and a concordance among sgRNA per gene of $100 \%$. Singleton hits represent significantly enriched genes with $\log _{2}$ fold change $\geq 2$, an adjusted sgRNA count of 1 and average control mean $\geq 100$ reads. Enriched hits not satisfying these criteria were classified into the unassigned group.

\section{Data availability.}

Raw data files for CRISPR screens have been deposited at GEO and can be found under the accession number GSE138343.

\section{Results}

\section{The MTOR and MAPK pathways are central components in resistance to sorafenib}

To identify genes whose loss-of-function variants contribute to resistance to sorafenib in AML, we selected MOLM13 cells, an AML cell line harboring an FLT3-ITD mutation resulting in sensitivity to several FLT3 inhibitors, including sorafenib. MOLM13 cells, engineered to express Cas9, were stably transduced with a genome-wide lentiviral sgRNA CRISPR knockout library ${ }^{33}$ and treated for 14 days with vehicle or $50 \mathrm{nM}$ sorafenib, a concentration projected to kill $80 \%$ of the cells within 3 days of drug administration $\left(\mathrm{IC}_{\text {» }}\right)$. Genomic DNA was harvested from control and sorafenib-treated cultures and evaluated for enriched sgRNA using $\mathrm{MAGeCK}$ robust rank aggregation (RRA) analyses ${ }^{34}$ (Figure 1A, B; Online Supplementary Table S1).

A comparison of sequencing reads from sorafenibtreated cultures and vehicle-treated controls identified significant enrichment for sgRNA targeting negative regulators of the MAPK and AKT/MTOR pathways (Figure 1B-D). The screen uncovered negative RAS/RAF/MEK/ERK regulator, leucine zipper like transcription regulator 1 (LZTR1), which inhibits the MAPK pathway by regulating RAS ubiquitination and degradation..$^{35,36}$ a negative regulator of RAS signaling, neurofibromin 1 (NF1); three components of the tuberous sclerosis (TSC) complex including TSC complex subunit 1 (TSC1), TSC complex subunit 2 (TSC2), and TBC1 domain family member 7 (TBC1D7). ${ }^{37}$ Top hits also included members of the GATOR1 complex, encoded by NPRL 2 and DEPCD $5{ }^{38}$ To prioritize candidates for validation, we developed a tiering structure that incorporates three key factors: evidence (determined by the number of sgRNA guide hits per gene), concordance (indicated by the agreement across the set of guides for a given gene) and discovery (based on effect size) to rank sgRNA hits and enable a progression to pathway analysis for lower scoring hits. ${ }^{38}$ Using the prioritization scheme, the tier 1 hits $(n=16)$ included LZTR1, TSC2, and TBC1D7 and several genes implicated in RNA splicing and ribosome biogenesis, such as DHX15, EBNA1BP2, LSM5, PUS7, RPSA and $A B C B 1$ transporter, linked to poor prognostic factors in AML (Online Supplementary Tables S1 and S2, Online Supplementary Figure S1). Our tier structure imposed additional constraints for ranking sgRNA hits into the more selective tiers, which generally preserved MAGeCK RRA rankings, although there were exceptions such as TSC1, which ranked as a tier 3 hit because of the variance in its log-fold change across the set of sgRNA for this gene. Using a false discovery rate cutoff, we decided to focus here on connecting the AKT/PI3K/MTOR and RAS/MAPK/MEK networks to verify candidates emerging from the screen (Figure 1B bottom panel, D).

\section{Deficiency of top hit genes decreases sensitivity to sorafenib in acute myeloid leukemia cell lines}

To validate top hit genes belonging to the LZTR1-connected network, we transduced MOLM13 cells with lentivirus expressing Cas9 and individual sgRNA to generate cells deficient in single genes. Sensitivity to sorafenib was assessed in $72 \mathrm{~h}$ cell viability MTS assays. Cells in which LZTR1, NF1, TSC1, TSC2, or NPRL2 were inactivated showed reduced sensitivity to sorafenib (Figure 1E). The degree of resistance to sorafenib varied across targeted genes, with TSC1- and LZTR1-deficient cells demonstrating the strongest resistance to sorafenib (parental IC $=5.03 \mathrm{nM}$, NT (non-targeting control) IC $=6.31 \mathrm{nM}$, sgTSC1 $\mathrm{IC}_{\mathrm{s}}=97.34 \mathrm{nM}$, and sgLZTR1 IC $=22.37 \mathrm{nM}$ ), while targeting of TSC2 yielded comparably more modest resistance to sorafenib $\left(\mathrm{IC}_{\mathrm{n}}=14 \mathrm{nM}\right.$ ) (Figure $1 \mathrm{E}$ ). TBC1D7deficient cells had decreased sensitivity to sorafenib while DEPCD5-deficient cells were modestly resistant (Online Supplementary Figure S2). Deficiencies of LZRT1, NF1, TSC1, TSC2 and NPRL2 were evident by western blot analysis (Online Supplementary Figure S3A). The corresponding efficiencies of CRISPR knockouts were determined using Inference of CRISPR Edits (ICE) software (Synthego.com) (Online Supplementary Figure S3B).

\section{Reduced expression levels of LZTR1, NF1, TSC1, and TSC2 correlate with reduced sensitivity to sorafenib in samples from patients with acute myeloid leukemia and deficiency results in hyperactivation of MAPK or MTOR pathways in acute myeloid leukemia cells}

We evaluated results from our CRISPR screen for relevance to drug sensitivity and gene expression profiles observed in patients' samples in the Beat AML database. ${ }^{3}$ RNA expression levels of LZTR1, NF1, and TSC2 showed negative correlations with sensitivity to sorafenib in samples from AML patients harboring FLT3-ITD mutations $(P<0.0001, P<0.001$, and $P<0.01$, respectively) (Figure $2 \mathrm{~A})$. We did not observe a significant negative correlation between gene expression and sensitivity to sorafenib for 
other screen hits, including RASA2, TBC1D7, NPRL2, and DEPDC5.

In MOLM13 cells, engineered to model LZTR1 and NF1 deficiencies, we observed elevated levels of phosphorylated ERK, suggesting increased activation of the MAPK signaling pathway (Figure 2B). As MAPK can cross-activate MTOR signaling, ${ }^{39-41}$ we observed increased phosphorylation level of MTORC1, similar to results with inactivated inhibitory functions of TSC1 and TSC2, indicating common aberrancy in downstream signaling (Figure 2B). Elevated levels of
A

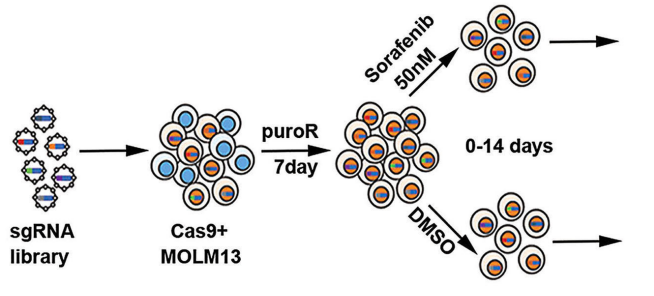

C
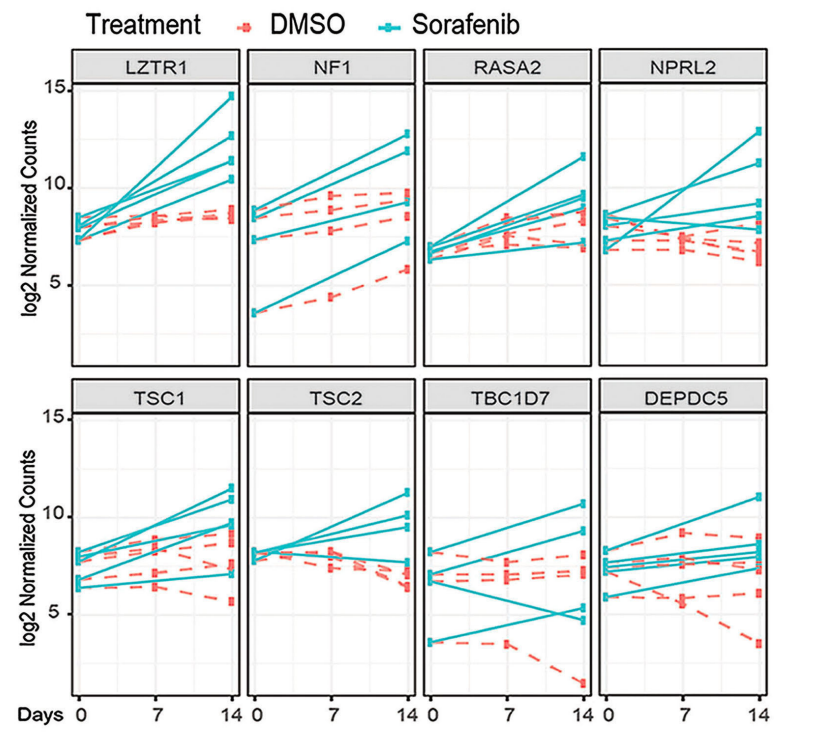

D

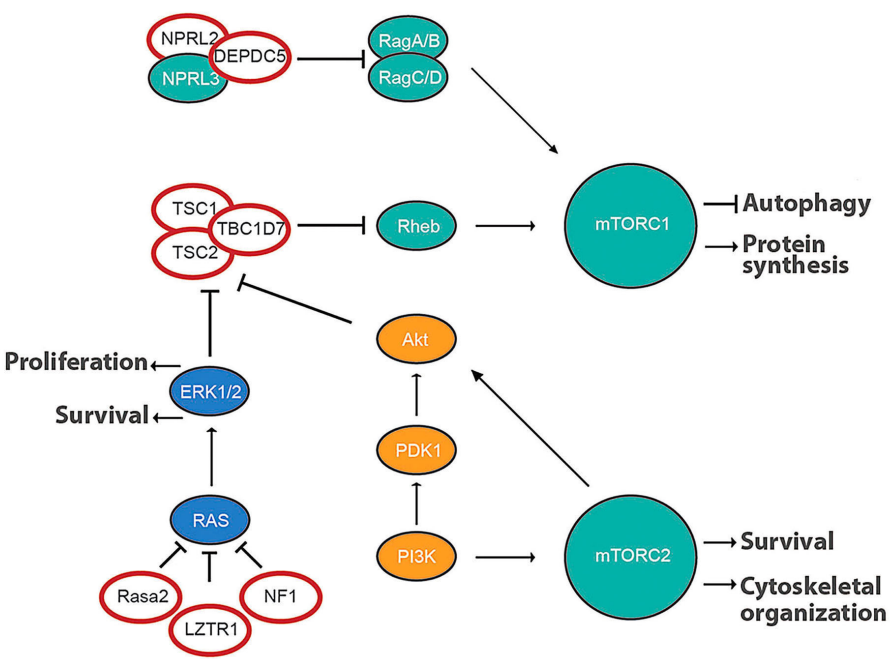

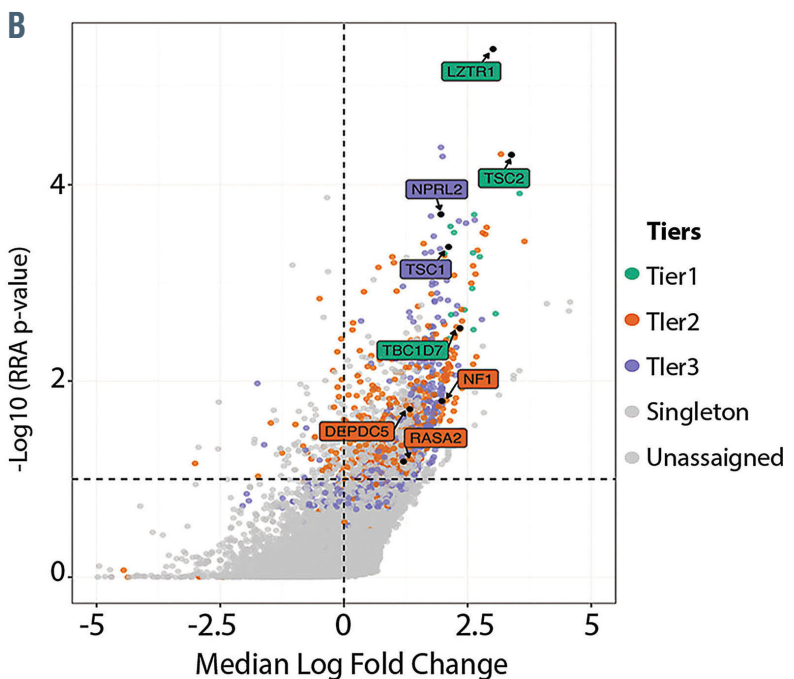

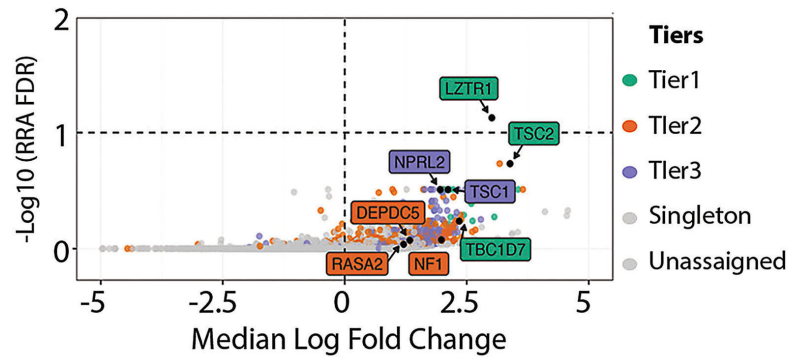

$\mathrm{E}$
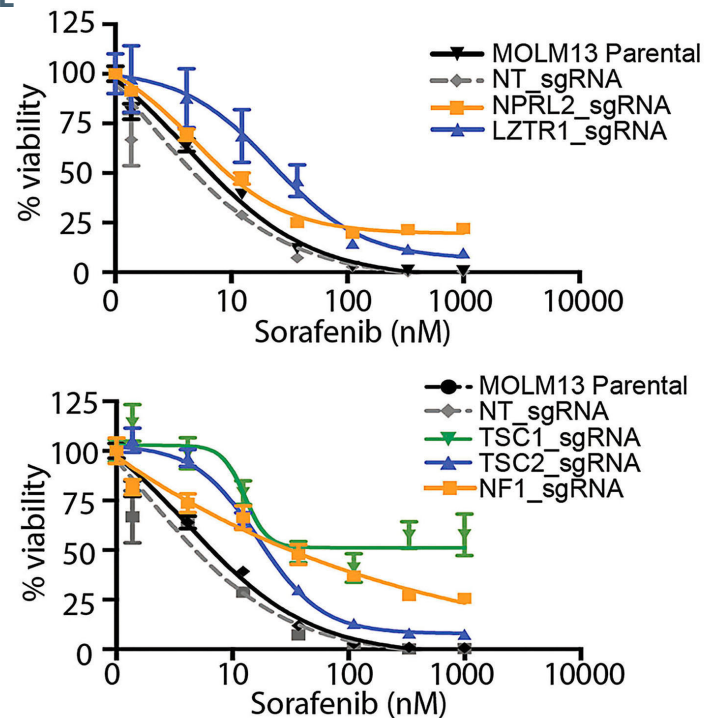

Figure 1. A genome-wide CRISPR knockout screen identifies negative regulators of MAPK and MTOR pathways, LZTR1, NF1, TSC1/2 and NPRL2, attenuating sensitivity to sorafenib in acute myeloid leukemia cell lines. (A) Schematic representation of the CRISPR knockout screen in MOLM13 acute myeloid leukemia cells. (B) Scatter plots of differential enrichment of single guide (sg)RNA in sorafenib-treated versus dimethylsulfoxide (DMSO)-treated control cells, analyzed at day 14 of drug exposure. Median log fold changes are plotted versus $P$-values (top panel), and false discovery rate (FDR)-adjusted $P$-values (bottom panel), generated from MAGeCK robust ranked aggregation (RRA) analysis. Colors represent various tiers associated with the ranking system based on directional concordance among sgRNA and significance of fold increase (see Methods section). (C) Plots of normalized sgRNA read counts targeting the five top candidate genes from sorafenib- and DMSOtreated samples over time. (D) Diagram of the MAPK and MTOR pathways with top hit genes shown in red circles. (E) Dose response curve of the $72 \mathrm{~h}$ sorafenib sensitivity assay performed on parental MOLM13 cells and MOLM13 cells transduced with sgRNA targeting LZTR1, NF1, TSC1, TSC2, NPRL2, and non-targeting (NT) control. The percentages of viable cells were measured using the MTS assay in triplicate with seven-point escalating drug concentrations. 
phospho-ERK were evident in TSC1-deficient, but not in TSC2-deficient cells, potentially reflecting different roles of TSC1 and TSC2 in the TSC complex. Levels of RAS protein were elevated in LZTR1-deficient cells, but not in those deficient in NF1 or TSC1 (Figure 2C).

\section{MTOR and MAPK pathways are upregulated in acute myeloid cells resistant to FLT3 inhibitors}

In a parallel approach to understand mechanisms of resistance to sorafenib, we generated AML cell lines resistant to FLT3 inhibitors by gradually exposing MOLM13 cells to type I (crenolanib) or type II (quizartinib and sorafenib) FLT3 inhibitors. Crenolanib- and sorafenib- resistant MOLM13 cells showed reduced sensitivity, detected by higher IC type I and type II inhibitors, whereas quizartinib-resistant cells showed resistance only to type II inhibitors (Figure 3A). To investigate whether there is an overlap between the acquisition of resistance by CRISPR-derived knockout cells versus resistance generated by prolonged exposure to drugs, we evaluated the activity of MTORC1 and MAPK pathways. We detected an increase in levels of phospho-ERK and elevated RAS levels in FLT3 drug-resistant cells, indicating upregulation of the MAPK pathway (Figure 3B). Surprisingly, levels of TSC2 were increased in crenolanib- and sorafenib-resis- tant cells; in contrast, loss of function for TSC2 was revealed in the CRISPR knockout resistance screen. Previous studies showed that the MAPK pathway can inhibit MTOR activity by phosphorylating TSC2 at S664 causing dissociation of the TSC complex observed in breast and colon carcinomas. ${ }^{41-43}$ This observation prompted us to test for levels of phospho-TSC2. We observed enhanced levels of phospho-TSC2 at S664 in these two cell lines, suggesting that inhibition of TSC complex formation by the MAPK pathway promotes resistance to FLT3 inhibitors. Moreover, we observed an elevated level of phospho-TSC2 at Y1571, which additionally impairs the TSC1-TSC2 interaction, ${ }^{44}$ supporting inactivation of TSC2 in these resistant cells, concordant with our CRISPR screen results.

\section{MEK inhibitors resensitize FLT3-inhibitor-resistant cell lines}

Data from the CRISPR screen and resistant cell lines suggested that upregulation of the MAPK signaling pathway contributes to resistance to FLT3 inhibitors in AML. This prompted us to hypothesize that inhibitors targeting the MAPK pathway may resensitize FLT3-inhibitorresistant cells. Parental and resistant cells were tested for sensitivity to the MEK inhibitor trametinib and MTOR inhibitors PP242, PI-103 and rapamycin. Resistant cells
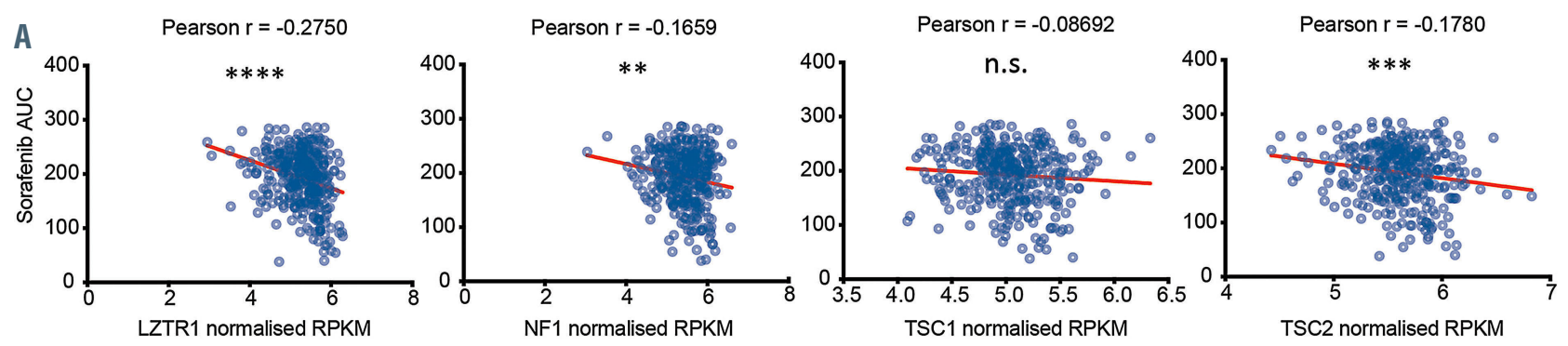

B
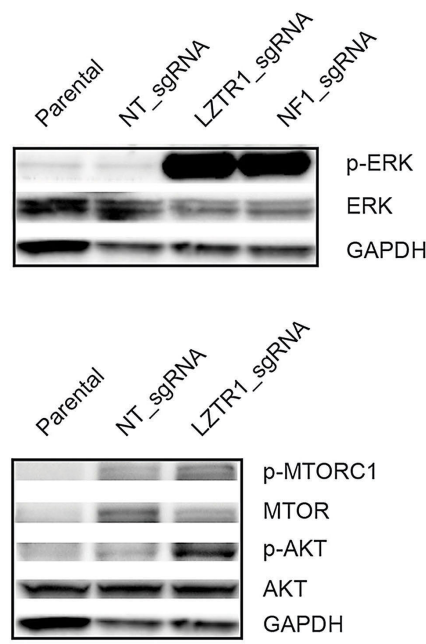
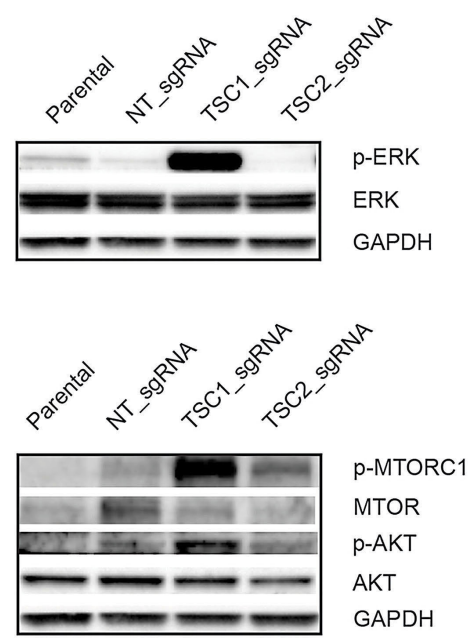

C
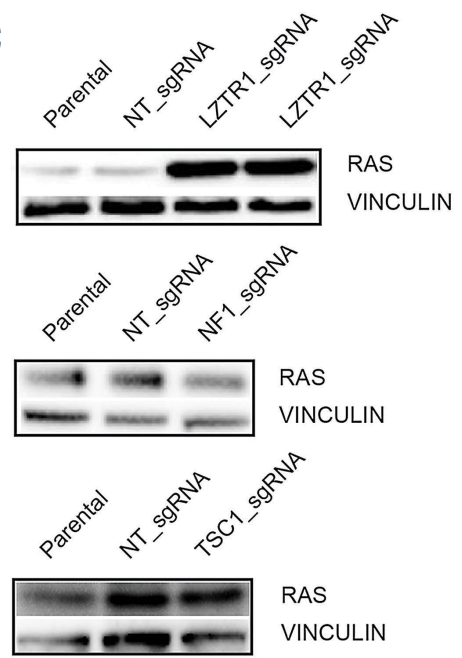

Figure 2. Low sensitivity to sorafenib correlates with low expression levels of CRISPR top hits LZTR1, NF1, and TSC2 in specimens from acute myeloid leukemia patients and inactivation in the MOLM13 acute myeloid leukemia cell line reveals aberrant activation of MAPK or MTOR pathways. (A) Scatter plots of RNA expression levels (shown as normalized reads per kilobase per million [RPKM]) plotted against drug sensitivity measured ex vivo as area under the curve (AUC) from samples from patients with acute myeloid leukemia harboring FLT3-ITD mutations $(n=86)$. Pearson correlation coefficients are shown as $(r)$ values Statistical significance is indicated. $* * * * P<0.0001, * * * P<0.001, * * P<0.01$, n.s., non-significant. (B, C) Immunoblot analyses of proteins from LZTR1-, NF1-, TSC1- and TSC2-deficient cells (LZTR1_sgRNA, NF1_sgRNA, TSC1_sgRNA and TSC2_sgRNA, correspondingly) in comparison to parental and non-targeting (NT_sgRNA) controls with indicated antibodies. p-ERK denotes phosphorylated-ERK (Thr202/Tyr204), p-MTOR denotes phosphorylated-MTOR at S2448. Vinculin and GAPDH immunoreactivity served as loading controls. 
showed greater sensitivity to trametinib relative to the parental cells (Figure 3C; Online Supplementary Figure $S 4 A$ ). Combinations of each FLT3 inhibitor with trametinib revealed enhanced efficacy in both resistant and parental AML cells (Figure 4A; Online Supplementary Figure $S 4 B$ ). Moreover, combinations of sorafenib and trametinib showed high synergy scores at several concentrations as analyzed by R_SynergyFinder ${ }^{45}$ (average synergy [zero interaction potency] score for parental cells $=3.449$ vs. 8.015 in sorafenib-resistant MOLM13 cells; synergy scores above 1 are significant). Similar synergy in sensitivity was obtained with trametinib in combination with either crenolanib or quizartinib (Online Supplementary Figure S4D). MTOR inhibitors PP242 and PI-103 did not exhibit substantial cell killing as single agents in any of the FLT3-inhibitor-resistant cell lines (Figure 3C, bottom panel; Online Supplementary Figure S4A) and a combination of MTOR inhibitor and a FLT3 inhibitor resulted in a marginal decrease in cell viability (Figure 4A bottom panels; Online Supplementary Figure $S 4 B, C)$. In contrast, MTOR inhibitors appeared to resensitize TSC1- and NPRL2-deficient cells to sorafenib (Online Supplementary Figure S5). The effect of PI-103 was more pronounced than that of PP242 which may reflect its dual targeting of MTOR and PI3K.

\section{Discussion}

Sorafenib, as well as other FLT3 inhibitors, in combination with standard-of-care chemotherapy prolongs the survival of AML patients with or without FLT3 mutations, although relapse caused by drug resistance remains a clinical challenge. To elucidate mechanisms of resistance to sorafenib, we subjected MOLM13 AML cells to genomewide CRISPR screening to identify genes whose loss-offunction contributes to reduced drug sensitivity. The top screen hits indicated that resistance to FLT3 inhibitors in AML can occur via aberrant activation of the AKT/PI3K/MTOR and RAS/MAPK signaling pathways. Our results are consistent with findings from previous studies on resistance to FLT3 inhibitors that revealed aberrant ERK and RAS signaling ${ }^{18,23}$ and extend these with the identification of a broad spectrum of genes regulating RAS/MAPK and, additionally, MTOR signaling pathways as modulators of resistance (Figure 4).

Analysis of the screen using the MAGeCK pipeline in combination with a tiering system developed previously ${ }^{32}$ identified LZTR1, TSC1/2, NPRL2, NF1, and TBC1D7 as significant hits. The identification of LZTR1 is not unexpected as LZTR1 loss confers MAPK activation by dysregulating RAS signaling; ${ }^{35,36}$ it also facilitates degradation of

A
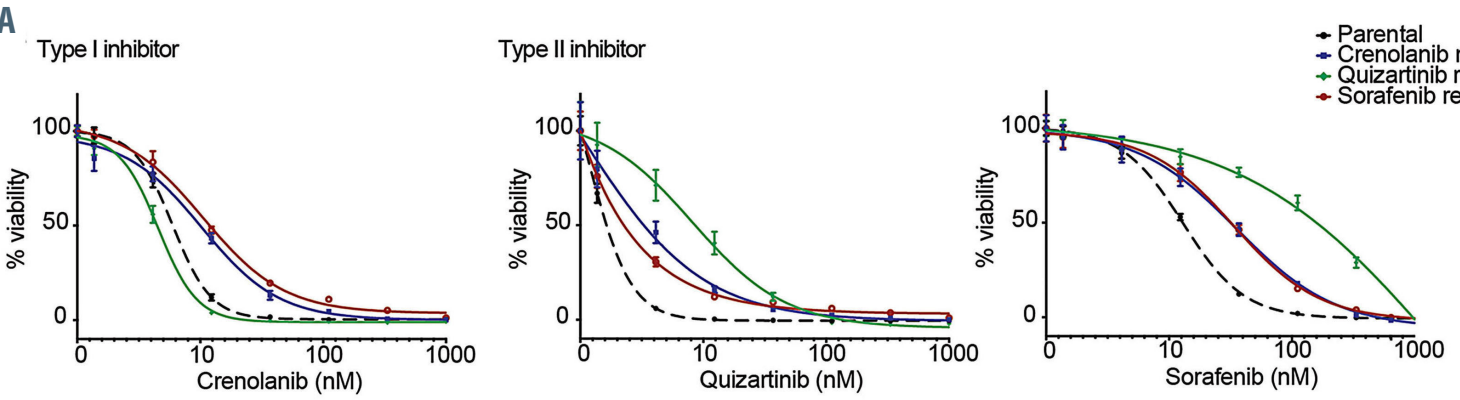

B
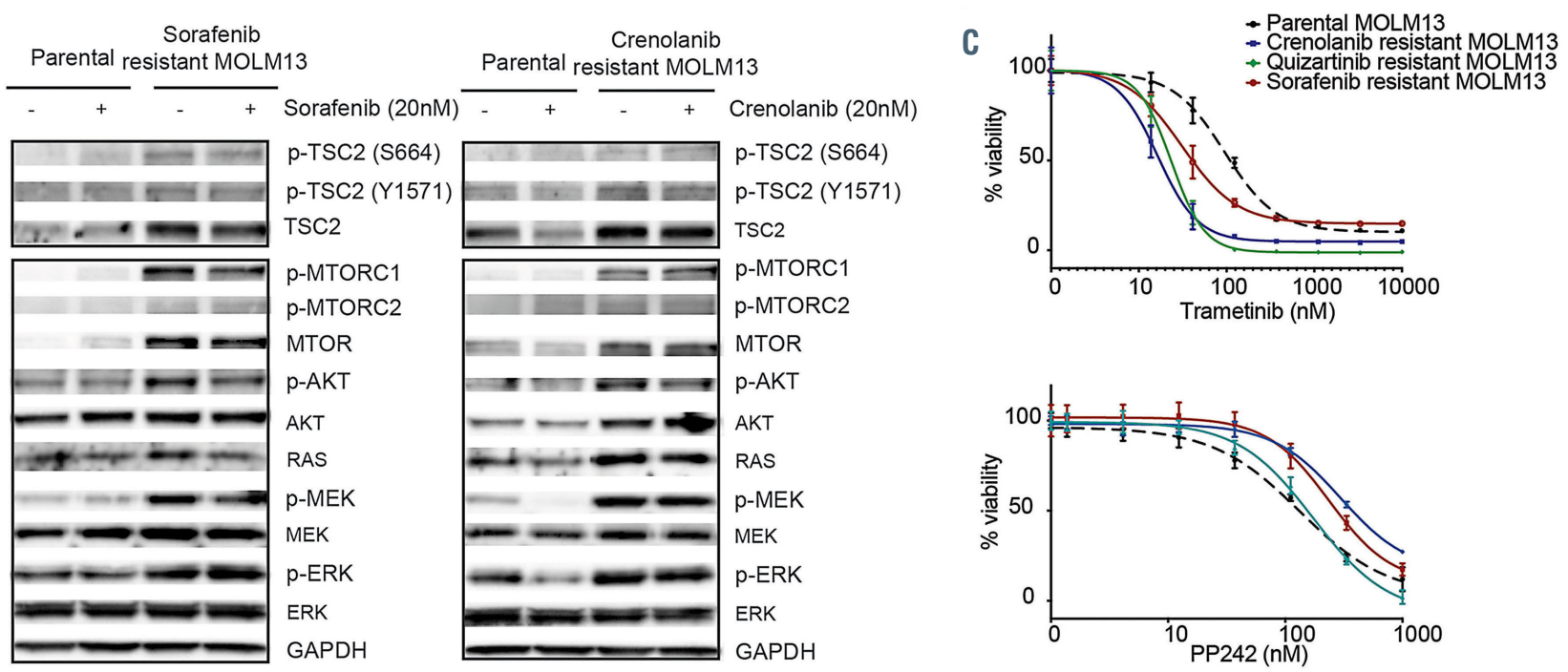

Figure 3. Acute myeloid leukemia cell lines made resistant to FLT3 inhibitors demonstrate activation of MAPK and/or MTOR pathways. (A) MOLM13 AML cell lines were made resistant to type I and type II FLT3 inhibitors by continuous exposure to crenolanib, quizartinib or sorafenib. Drug sensitivity assays were performed for $72 \mathrm{~h}$ on parental and FLT3-inhibitor-resistant MOLM13 cells. Cell viability was measured in triplicate using the MTS assay with seven-point escalating drug concentrations. (B) Immunoblot of whole cell lysates from parental MOLM13 and MOLM13 cells resistant to crenolanib and sorafenib treated with dimethlysulfoxide or FLT3 inhibitors at $20 \mathrm{nM}$ concentration for $4 \mathrm{~h}$ performed with indicated antibodies. (C) Dose response curves of trametinib and PP242 on parental and FLT3-inhibitorresistant MOLM13 cells. 
RAS-GTPases. ${ }^{46,47}$ Consistent with our results, loss of LZTR1 function has recently been shown to cause resistance to tyrosine kinase inhibitors including several FLT3 inhibitors (tandutinib, quizartinib, and ponatinib) in AML cell lines. ${ }^{36}$ LZTR1 loss-of-function mutations have been observed in other cancers including glioblastoma multiforme, adrenocortical cancer, and pancreatic cancer, and have also recently been reported in hepatocellular carcinoma, a cancer for which sorafenib is a first-line therapy. ${ }^{48,49}$ The screen hits included components of the TSC and the GATOR complexes, which have not been previously identified as modulators of resistance to FLT3 inhibitors. Our screen also identified two negative regulators of RAS: NF1 and RASA2. Loss-of-function mutations in NF1 have been associated with poor prognosis in AML patients, ${ }^{50}$ suggest- ing that patients with NF1 mutations would have poor sensitivity to FLT3 inhibitors.

TSC2 and TBC1D7 along with TSC1 form the TSC complex, which acts as a GTPase activating protein for RHEB, a small G-protein upstream of MTOR complex 1 (MTORC1). ${ }^{36,51-53}$ MTORC1 is activated by another small G-protein, RAG, which is negatively regulated by the GATOR1 complex, comprising NPRL2, NPRL3, and DEPDC5 proteins. ${ }^{37}$ Our screen identified TSC1 and components of the GATOR1 complex, DEPDC5 and NPRL2. Loss-of-function variants of TSC1 and TSC2 are found in $\sim 16 \%$ of patients with hepatocarcinoma and are associated with an aggressive form of this malignancy. ${ }^{54}$ Our data suggest that the roles of TSC1 and TSC2 are complex; we note that TSC1-, but not TSC2-deficient MOLM13 cells
A

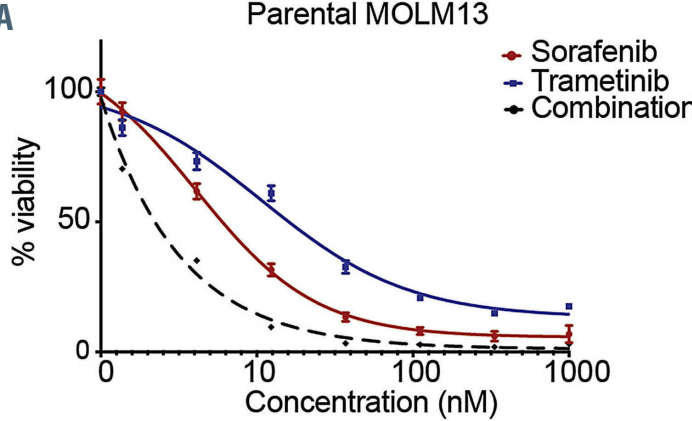

Parental MOLM13

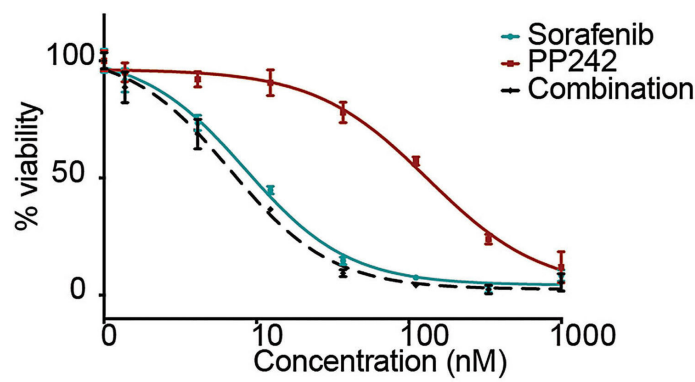

Sorafenib resistant MOLM13

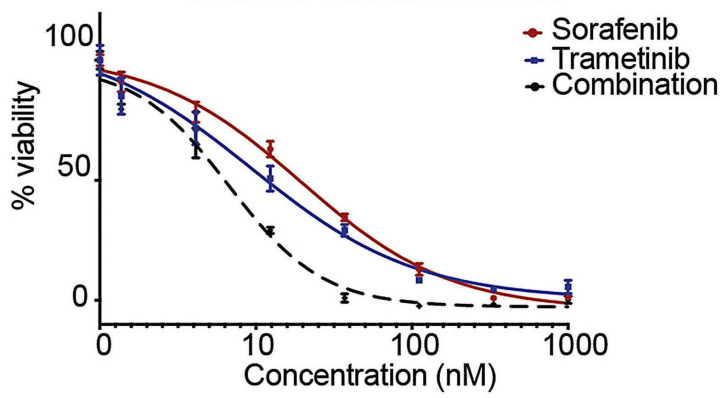

Sorafenib resistant MOLM13

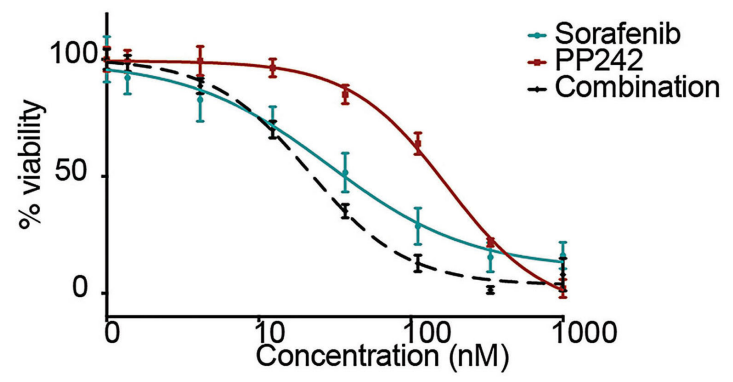

B

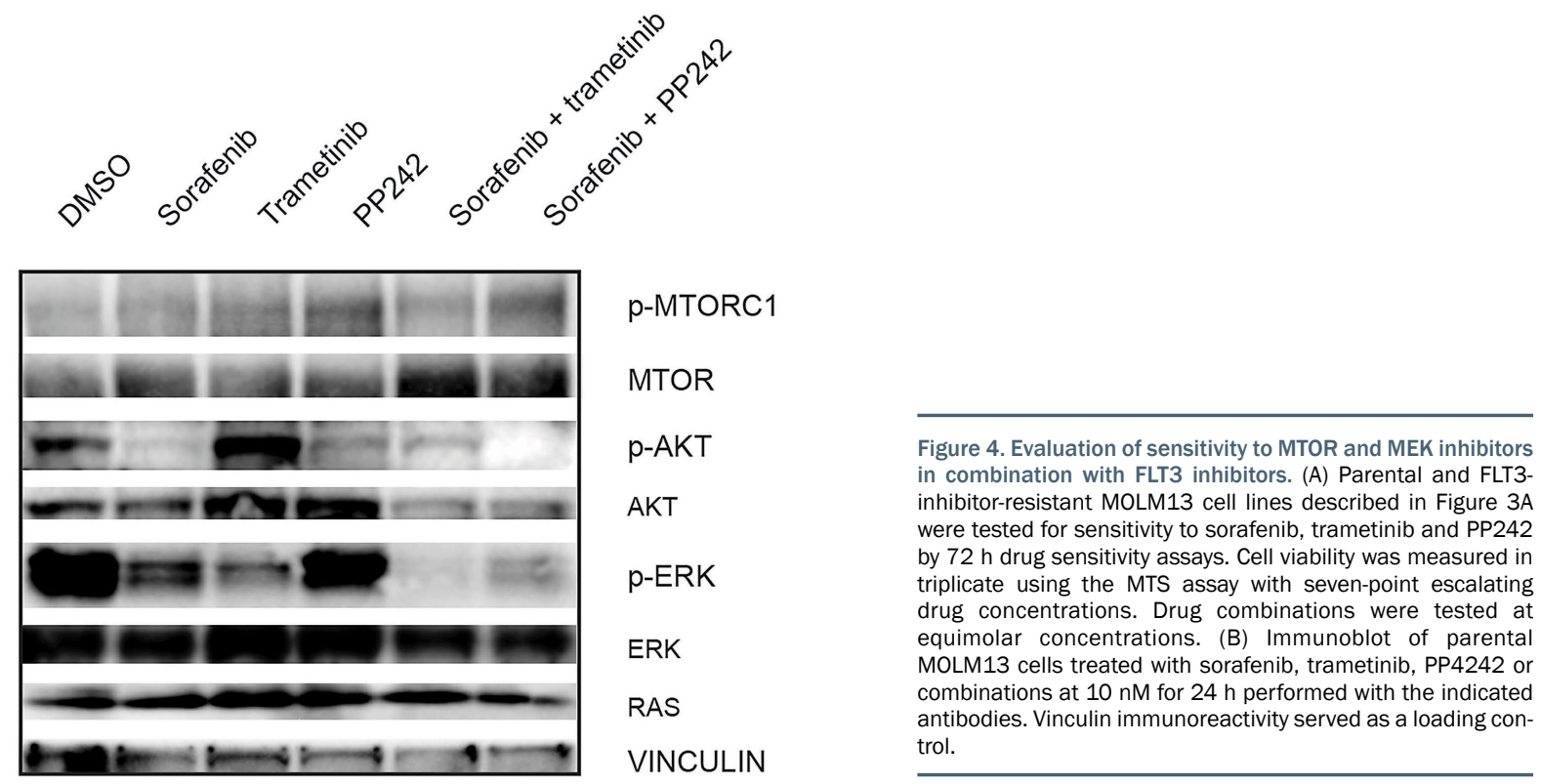


showed increased phospho-ERK activity. This observation may not be surprising given the structural difference between the two proteins; TSC1 lacks the kinase domain that is present in TSC2. ${ }^{36,51}$ It is possible that TSC1 executes functions outside of its interactions with TSC2 to regulate MAPK signaling. Consistent with results from the CRISPR screen, samples from AML patients harboring FLT3-ITD mutations with reduced RNA expression levels of LZTR1, NF1, and TSC2 exhibited less sensitivity to sorafenib.

Upregulation of phospho-MTORC1 (Ser2481) is observed in two FLT3-inhibitor-resistant cell lines, made by gradual exposure to higher concentrations of FLT3 inhibitors, supporting a role for MTORC1 in resistance to FLT3 inhibitors. Increased phospho-ERK levels in FLT3resistant cells confirmed the importance of the MAPK pathway in FLT3 inhibitor resistance. Concordantly, the resistant cells demonstrated enhanced sensitivity to MEK inhibitors. We also showed that the connection between MAPK and MTOR pathways influenced FLT3 inhibitor resistance. We found enhanced levels of phospho-TSC2 at S664 and Y1571 in the resistant lines which are regulated by phosphorylated ERK and AKT, respectively. These phosphorylation events inhibit the formation of the TSC complex, mimicking TSC1 and TCS2 loss-of-function hits as revealed by the CRISPR resistance screen. The combination of FLT3 plus MEK inhibitors has synergistic effectiveness in both sensitive and resistant cells, a finding that is consistent with data from a recent study that demonstrated synergy for the combination of crenolanib with trametinib in Ba/F3 cells harboring PTPN11 A72D, FLT3 D835Y or double mutations of both. ${ }^{22}$ Similarly, the combination of sorafenib or pazopanib with trametinib showed strong synergy in MOLM13 cells. ${ }^{25}$ Moreover, the combination of sorafenib with the MEK inhibitor, PD0325901, showed synergy in MOLM14 and MV4;11 AML cell lines, ${ }^{23,55}$ and the combination of gilteritinib and trametinib had enhanced efficacy in MOLM14 cells with NRAS G12C and NRAS Q61K. ${ }^{18}$ Enhanced efficacy was observed in AML patients' samples assayed ex vivo for sensitivity to a combination of an FLT3 inhibitor (quizartinib) and trametinib (Beat AML data, Online Supplementary Figure S4E). Evaluation of signaling in FLT3i-resistant cells and top hits from the CRISPR knockout screen underscore activation of the MTOR pathway in sorafenib resistance. Assessment of sensitivity to an MTOR inhibitor in combination with an FLT3 inhibitor showed a profound effect only in TSC1- and NPRL2-deficient cells. This result may indicate reliance on multiple pathways in FLT3-inhibitor-resistant cells.

Increased activity of JAK/STAT5 has also been implicated in resistance to FLT3 inhibitors. Granulocytemacrophage colony-stimulating factor and interleukin-3 mediate FLT3 resistance in AML cells via JAK/STAT5 and PIM/cytokine-activated JAK/STAT5 signaling. ${ }^{26}$ Although, we did not evaluate JAK/STAT5 pathways in this study, our CRISPR screen did identify several negative regulators of JAK/STAT5 pathways, including PTPN1, SUMO3, and PTPN6. Additionally, our screen identified several tier 1 hits involved in RNA metabolism and splicing, including DHX15, EBNA1BP2, LSMR, PUS7 and RPSA. DHX15, for example, encodes an RNA helicase that is commonly mutated in AML patients with RUNX1RUNX1T1 fusions. ${ }^{56}$ It will be important to pursue these findings in subsequent studies given the roles of RNA metabolism in cancer pathogenesis. ${ }^{57.59}$

\section{Disclosures}

JWT has received research support from Agios, Aptose, Array, AstraZeneca, Constellation, Genentech, Gilead, Incyte, Janssen, Petra, Seattle Genetics, Syros, and Takeda. The other authors have no conflicts of interest to disclose.

\section{Contributions}

$A D$ and $T N$ performed experimentation, $D B$ and $C E$ performed data analyses, $A D, S K, T N$, and JT, wrote the manuscript, JT, SM, and TN supervised the study.

\section{Acknowledgments}

We thank the OHSU Massively Parallel Sequencing Shared Resource and Flow Cytometry Core for technical support, and Sunil K. Joshi for reviewing the manuscript.

\section{Funding}

This study was supported by grants from the National Cancer Institute (1U01CA217862, 1U54CA224019, 3P30CA06953318S5). JWT received grants from the $V$ Foundation for Cancer Research, the Gabrielle's Angel Foundation for Cancer Research, and the National Cancer Institute (1R01CA183947). TN is supported by grant R50 CA251708-01.

\section{References}

1. Papaemmanuil E, Gerstung M, Bullinger L, et al. Genomic classification and prognosis in acute myeloid leukemia. N Engl J Med. 2016;374(23):2209-2221.

2. Döhner H, Weisdorf DJ, Bloomfield CD. Acute myeloid leukemia. N Engl J Med. 2015;373(12):1136-1152.

3. Tyner JW, Tognon CT, Bottomly D, et al. Functional genomic landscape of acute myeloid leukaemia. Nature. 2018;562(7728): 526-531.

4. McCurdy SR, Levis MJ. Emerging molecular predictive and prognostic factors in acute myeloid leukemia. Leuk Lymphoma. 2018; 59(9):2021-2039.

5. Cancer Genome Atlas Research Network. Genomic and epigenomic landscapes of adult de novo acute myeloid leukemia. $\mathrm{N}$ Engl J Med. 2013;368(22):2059-2074.
6. Gilliland DG, Griffin JD. The roles of FLT3 in hematopoiesis and leukemia. Blood. 2002;100(5):1532-1542.

7. Daver N, Schlenk RF, Russel NH, et al. Targeting FLT3 mutations in AML: review of current knowledge and evidence. Leukemia. 2019;33(2):299-312

8. Small D. FLT3 mutations: biology and treatment. Hematology Am Soc Hematol Educ Program. 2006(1):178-184.

9. Nakao M, Yokota S, Iwai T, et al. Internal tandem duplication of the flt 3 gene found in acute myeloid leukemia. Leukemia. 1996;10(12):1911-918.

10. Yokota S, Kiyoi H, Nakao M, et al. Internal tandem duplication of the FLT3 gene is preferentially seen in acute myeloid leukemia and myelodysplastic syndrome among various hematological malignancies. A study on a large series of patients and cell lines. Leukemia. 1997;11(10):1605-1609.
11. Tse KF, Mukheriee G, Small D. Constitutive activation of FLT3 stimulates multiple intracellular signal transducers and results in transformation. Leukemia. 2000;14(10): 1766-1776.

12. Yamamoto Y, Nakano Y, Suzuki R, et al. Activating mutation of D835 within the activation loop of FLT3 in human hematologic malignancies. Blood. 2001;97(8):2434-2439.

13. Kiyoi H, Yanada M, Ozekia K. Clinical significance of FLT3 in leukemia. Int J Hematol. 2005;82(2):85-92.

14. Larrosa-Garcia M, Baer MR. FLT3 inhibitors in acute myeloid leukemia: current status and future directions. Mol Cancer Ther 2017;16(6):991-1001.

15. Wu M, Li C, Zhu X. FLT3 inhibitors in acute myeloid leukemia. J Hematol Oncol. 2018;11(1):133.

16. Stone RM, Mandrekar S, Sanford SL, et al. Midostaurin plus chemotherapy for acute 
myeloid leukemia with a FLT3 mutation. N Engl J Med. 2017;377(5):454-464.

17. Perl AE, Altman JK, Cortes J, et al. Selective inhibition of FLT3 by gilteritinib in relapsed or refractory acute myeloid leukaemia: a multicentre, first-in-human, open-label, phase 1-2 study. Lancet Oncol. 2017;18(8): 1061-1075

18. McMahon CM, Ferng T, Canaani J, et al. Clonal selection with Ras pathway activation mediates secondary clinical resistance to selective FLT3 inhibition in acute myeloid leukemia. Cancer Discov. 2019;9(8):10501063.

19. Smith CC, Wang Q, Chin C-S, et al. Validation of ITD mutations in FLT3 as a therapeutic target in human acute myeloid leukaemia. Nature. 2012:485(7397):260-263.

20. Man CH, Fing TK, Ho C, et al. Sorafenib treatment of FLT3-ITD(+) acute myeloid leukemia: favorable initial outcome and mechanisms of subsequent nonresponsiveness associated with the emergence of a D835 mutation. Blood. 2012;119(22):51335143.

21. Albers $C$, Leischner $H$, Verbeek $M$, et al. The secondary FLT3-ITD F691L mutation induces resistance to AC220 in FLT3-ITD+ AML but retains in vitro sensitivity to PKC412 and Sunitinib. Leukemia. 2013;27 (6):1416-1418.

22. Zhang H, Savage S, Schultz AR, et al. Clinical resistance to crenolanib in acute myeloid leukemia due to diverse molecular mechanisms. Nature Commun. 2019;10(1): 244-257.

23. Bruner JK, Ma HS, Li L, et al. Adaptation to TKI treatment reactivates ERK signaling in tyrosine kinase-driven leukemias and other malignancies. Cancer Res. 2017;77(20):55545563.

24. Yang X, Sexauer A, Levis M. Bone marrow stroma-mediated resistance to FLT3 inhibitors in FLT3-ITD AML is mediated by persistent activation of extracellular regulated kinase. Br J Haematol. 2014;164(1):61-72.

25. Martínez-López J, Linares M, Morales ML, et al. Preclinical evidence that trametinib enhances the response to tyrosine kinase inhibitors in acute myeloid leukemia. Blood. 2016;128(22):1581.

26. Sung PJ, Sugita M, Koblish $H$, et al. Hematopoietic cytokines mediate resistance to targeted therapy in FLT3-ITD acute myeloid leukemia. Blood Adv. 2019;3(7): 1061-1072.

27. Lindblad $O$, Cordero E, Puissant $A$, et al. Aberrant activation of the PIBK/mTOR pathway promotes resistance to sorafenib in AML. Oncogene. 2016:35(39):5119-5131.

28. Wilhelm S, Carter C, Lynch $M$, et al. Discovery and development of sorafenib: a multikinase inhibitor for treating cancer. Nat Rev Drug Discov. 2006;5(10):835-844.

29. Ravandi F, Alattar ML, Grunwald MR, et al. Phase 2 study of azacytidine plus sorafenib in patients with acute myeloid leukemia and
FLT-3 internal tandem duplication mutation. Blood. 2013;121(23):4655-4662

30. Röllig C, Serve H, Huttmann A, et al Addition of sorafenib versus placebo to standard therapy in patients aged 60 years or younger with newly diagnosed acute myeloid leukaemia (SORAML): a multicentre, phase 2, randomised controlled trial. Lancet Oncol. 2015;16(16):1691-1699.

31. Chen Y-B, Li S, Lane AA, et al. Phase I trial of maintenance sorafenib after allogeneic hematopoietic stem cell transplantation for Fms-like tyrosine kinase 3 internal tandem duplication acute myeloid leukemia. Biol Blood Marrow Transplant. 2014;20(12): 2042-2048.

32. Nechiporuk T, Kurtz SE, Nikolova O, et al. The TP53 apoptotic network is a primary mediator of resistance to BCL2 inhibition in AML cells. Cancer Discov. 2019;9(7):910925.

33. Tzelepis K, Koike-Yusa H, De Braekeleer E, et al. A CRISPR dropout screen identifies genetic vulnerabilities and therapeutic targets in acute myeloid leukemia. Cell Rep. 2016; 17(4):1193-1205.

34. Li W, Xu H, Xiao T, et al. MAGeCK enables robust identification of essential genes from genome-scale CRISPR/Cas9 knockout screens. Genome Biol. 2014;15(12):554-566.

35. Steklov M, Pandolfi S, Baietti MF, et al, Mutations in LZTR1 drive human disease by dysregulating RAS ubiquitination. Science. 2018;362(6419):1177-1182.

36. Bigenzahn J, Collu GM, Kartnig F, et al. LZTR1 is a regulator of RAS ubiquitination and signaling. Science. 2018;362(6419):11711177 .

37. Dibble CC, Elis W, Menon S, et al. TBC1D7 is a third subunit of the TSC1-TSC2 complex upstream of mTORC1. Mol Cell. 2012;47(4):535-546

38. Shen K, Huang RK, Brignole RG, et al Architecture of the human GATOR1 and GATOR1-Rag GTPases complexes. Nature. 2018:556(7699):64-69.

39. Carriere A, Romea Y, Acosta-Jacquez JK, et al. ERK1/2 phosphorylate Raptor to promote Ras-dependent activation of mTOR complex 1 (mTORC1). J Biol Chem. 2011;286(1):567-577.

40. Mendoza MC, Er EE, Blenis J. The Ras-ERK and PI3K-mTOR pathways: cross-talk and compensation. Trends Biochem Sci. 2011;36 (6):320-328.

41. Ma L, Chen Z, Erdjument-Bromage $\mathrm{H}$, et al. Phosphorylation and functional inactivation of TSC2 by Erk: implications for tuberous sclerosis and cancer pathogenesis. Cell. 2005;121(2):179-193

42. Ma L, Teruya-Feldstein J, Bonner P, et al. Identification of S664 TSC2 phosphorylation as a marker for extracellular signal-regulated kinase-mediated mTOR activation in tuberous sclerosis and human cancer. Cancer Res. 2007;67(15):7106-7112

43. Ballif BA, Roux PP, Gerber SA, et al.
Quantitative phosphorylation profiling of the ERK/p90 ribosomal S6 kinase-signaling cassette and its targets, the tuberous sclerosis tumor suppressors. Proc Natl Acad Sci U S A. 2005;102(3):667-672.

44. Aicher LD, Campbell JS, Yeung RS. Tuberin phosphorylation regulates its interaction with hamartin: two proteins involved in tuberous sclerosis. J Biol Chem. 2001;276 (24):21017-21021.

45. He L, Kulesskiy E, Saarela J, et al. Methods for high-throughput drug combination screening and synergy scoring. Methods Mol Biol. 2018;1711:351-398.

46. Castel P, Cheng A, Cuevas-Navarro A, et al. RIT1 oncoproteins escape LZTR1-mediated proteolysis. Science. 2019; 363(6432):12261230.

47. Abe T, Umeki I, Kanno S-I, et al. LZTR1 facilitates polyubiquitination and degradation of RAS-GTPases. Cell Death Differ. 2020;27(3):1023-1035

48. Cancer Genome Atlas Research Network Comprehensive and integrative genomic characterization of hepatocellular carcinoma. Cell. 2017;169(7):1327-1341.

49. Frattini V, Trifonov, V, Chan JM, et al. The integrated landscape of driver genomic alterations in glioblastoma. Nat Genet. 2013;45 (10): 1141-1149.

50. Eisfeld A-K, Kohlschmidt J, Mrozek K, et al. NF1 mutations are recurrent in adult acute myeloid leukemia and confer poor outcome. Leukemia. 2018;32(12):2536-2545.

51. Huang J, Manning BD. The TSC1-TSC2 complex: a molecular switchboard controlling cell growth. Biochem J. 2008;412(2):179190

52. Laplante M, Sabatini DM. mTOR signaling in growth control and disease. Cell. 2012;149(2):274-293.

53. Manning $\mathrm{BD}$, Toker A. AKT/PKB signaling: navigating the network. Cell. 2017;169(3): 381-405.

54. Ho DWH, Chan LK, Chui YT, et al. TSC1/2 mutations define a molecular subset of HCC with aggressive behaviour and treatment implication. Gut. 2017;66(8):1496-1506.

55. Morales ML, Arenas A, Ortiz-Ruiz A, et al. $M E K$ inhibition enhances the response to tyrosine kinase inhibitors in acute myeloid leukemia. Sci Rep. 2019;9(1):18630-18641.

56. Opatz S, Bamopoulus SA, Metzeler KH, et al. The clinical mutatome of core binding factor leukemia. Leukemia. 2020;34(6): 1553-1562.

57. Wang BD, Lee NH. Aberrant RNA splicing in cancer and drug resistance. Cancers (Basel). 2018;10(11):458-482.

58. Desterro J, Bak-Gordon P, Carmo-Fonseca $M$. Targeting mRNA processing as an anticancer strategy. Nat Rev Drug Discov. 2020;19(2):112-129.

59. Di C, Syafrizayanti, Zhang $Q$, et al Function, clinical application, and strategies of pre-mRNA splicing in cancer. Cell Death Differ. 2019;26(7):1181-1194. 